\title{
экология животных
}

УДК $591.9(262.81)$

\section{ВИДОВОЙ СОСТАВ И ЗООГЕОГРАФИЧЕСКАЯ ХАРАКТЕРИСТИКА ПАНЦИРНЫХ КЛЕЩЕЙ (ACARIFORMES, ОRIBATIDA) ПОБЕРЕЖЬЯ И ОСТРОВОВ СЕВЕРО-ЗАПАДНОЙ ЧАСТИ КАСПИЙСКОГО МОРЯ}

\author{
๑ 2013 Абдурахманов Г.М. ${ }^{1}$, Грикурова А.А.ㄹ, Субиас Л.С. ${ }^{3}$, Штанчаева У.Я. ${ }^{3}$, Курамагомедов Б.М. ${ }^{1}$ \\ ${ }^{1}$ Дагестанский государственный университет \\ 2 Прикаспийский институт биологических ресурсов Дагестанского научного центра РАН
}

${ }^{3}$ Мадридский университет Комплутенсе

\begin{abstract}
В статье приводятся результаты исследования панцирных клещей побережья и островов северо-западной части Каспийского моря - обновленный видовой состав и данные об ареалогии видов.

The article presents the results of the study of oribatid mites of coastal and of islands of the north-west part of the Caspian sea the updated list of species, data about distribution of species.

Ключевые слова: панцирные клещи, прибрежные экосистемы, острова, Каспийское море, видовой состав, распространение.

Keywords: the oribatid mites, coastal ecosystems, islands, Caspian Sea, list of species, distribution.
\end{abstract}

В ходе комплексных экспедиций с целью выяснения биологического разнообразия были обследованы различные биотопы островов северо-западной части Каспийского моря (о-ва Чечень, Тюлений, Нордовый) и его прибрежной части.

Нами выявлено видовое разнообразие панцирных клещей исследуемой территории [1; 2]. К настоящему времени эти данные дополнены, полный список видов приведен ниже (табл. 1). Всего было обследовано 32 биотопа с различными типами растительности, в том числе 11 местообитаний, находящихся на побережье, и 21 островной биотоп.

Исследуемая территория представлена Приморским флористическим районом Дагестана [3] и характеризуется литоральной песчаной и пустынной растительностью с солянково-полынными комплексами [4], а также болотными ландшафтами с тростниково-рогозовыми ассоциациями и плавнями, которые встречаются в дельтах рек и на островах.

Таблица 1

Видовой состав орибатид прибрежных экосистем и островов северо-западной части Каспийского моря

\begin{tabular}{|c|c|c|c|c|c|c|}
\hline \multirow[b]{2}{*}{ № } & \multirow[b]{2}{*}{ Виды панцирных клещей } & \multicolumn{2}{|c|}{$\begin{array}{c}\text { Прибрежные } \\
\text { экосистемы } \\
\end{array}$} & \multicolumn{3}{|c|}{$\begin{array}{c}\text { Островные } \\
\text { экосистемы }\end{array}$} \\
\hline & & 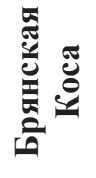 & 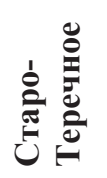 & 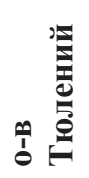 & 亮 & 苞 \\
\hline 1 & Aphelacarus acarinus (Berlese, 1910) & + & & & & \\
\hline 2 & Haplochthonius (H.) sanctaeluciae Bernini, $1973^{\text {К Д }}$ & + & & & & \\
\hline 3 & Sphaerochthonius pallidus Muñoz-Mingarro, 1987 К д & + & & & & \\
\hline 4 & Sphaerochthonius splendidus (Berlese,1904) & & + & & & \\
\hline 5 & Epilohmannia cylindrica cylindrica (Berlese, 1904) & + & + & & & \\
\hline 6 & Epilohmannia cylindrica minima Schuster, 1960 & & & & & + \\
\hline 7 & Papillacarus pseudoaciculatus Mahunka, $1980{ }^{K} \mathrm{~L}$ & + & & & & \\
\hline 8 & Neoliodes ionicus Sellnick, $1931^{К ~ Д ~}$ & + & & & & \\
\hline
\end{tabular}




\begin{tabular}{|c|c|c|c|c|c|c|}
\hline 9 & Pyroppia tajikistanica Krivolutsky et Christov, 1970 & & + & & & \\
\hline 10 & Ramusella (R.) puertomonttensis Hammer, 1962 & + & & + & & \\
\hline 11 & Ramusella (Rectoppia) strinatii (Mahunka, 1980) ${ }^{d}$ & & + & & & \\
\hline 12 & Discoppia (Cylindroppia) cylindrica (Pérez-Íñigo, 1965) & + & & + & & \\
\hline 13 & Microppia minus minus (Paoli, 1908) & & & + & & \\
\hline 14 & Lauroppia similifallax Subías et Mínguez, $1986^{\text {म }}$ & & & + & & \\
\hline 15 & Oppiella (O.) nova nova (Oudemans, 1902) & & & + & & \\
\hline 16 & Subiasella (Lalmoppia) subiasi (Mahunka, 1987) ${ }^{\mathrm{K}} \mathrm{d}$ & & + & & & \\
\hline 17 & Suctobelbella (S.) acutidens duplex (Strenzke, 1950) & & & + & & \\
\hline 18 & Suctobelbella (S.) subcornigera subcornigera (Forsslund, 1941) & + & & & & \\
\hline 19 & Suctobelbella (Flagrosuctobelba) nasalis (Forsslund, 1941) & & & + & & \\
\hline 20 & Tectocepheus velatus sarekensis Trägårdh, 1910 & + & & + & & \\
\hline 21 & Scutovertex sculptus Michael, 1879 & + & + & & & \\
\hline 22 & $\begin{array}{l}\text { Bipassalozetes (Passalobates) linearis (Higgins et Woolley, } \\
1962) \mathrm{Kд}\end{array}$ & & + & + & & + \\
\hline 23 & Passalozetes africanus Grandjean, 1932 & + & + & + & & \\
\hline 24 & Berlesezetes ornatissimus (Berlese, 1913) ${ }^{\lambda}$ & & + & & & \\
\hline 25 & Oribatella (Oribatella) reticulata Berlese, 1916 & & + & & & \\
\hline 26 & Oribatella $(O$.$) tridactyla Ruiz, Subías et Kahwash, 1991^{\mathrm{Kd}}$ & + & & & & \\
\hline 27 & Tectoribates ornatus (Schuster, 1958) & + & & & & \\
\hline 28 & Trichoribates (Trichoribates) novus (Sellnick,1928) & & + & & & \\
\hline 29 & Trichoribates (Latilamellobates) clavatus Mihelčič, 1956 Кд & & & + & & \\
\hline 30 & Trichoribates (Latilamellobates) naltschicki (Shaldybina, 1971) & + & + & & & + \\
\hline 31 & Zetomimus (Protozetomimus) acutirostris (Mihelčič, 1957) & & & & + & \\
\hline 32 & Chamobates (Xiphobates) rastratus (Hull, 1914) & & & + & & \\
\hline 33 & Punctoribates (Punctoribates) punctum (Koch,1839) & & + & & & + \\
\hline 34 & Punctoribates (Minguezetes) hexagonus Berlese, 1908 & + & + & & & \\
\hline 35 & Podoribates longipes (Berlese, 1887) ${ }^{\mathrm{A}}$ & + & & & & \\
\hline 36 & Oribatula (Oribatula) interrupta interrupta (Willmann, 1939) & + & & & & \\
\hline 37 & Oribatula (O.) tibialis saljanica Kulijev, $1962^{Д}$ & & & + & & \\
\hline 38 & $\begin{array}{l}\text { Oribatula (Zygoribatula) caspica Shtanchaeva, Grikurova, } \\
\text { Subias, } 2011^{\text {KД*** }}\end{array}$ & + & + & + & & + \\
\hline 39 & Oribatula (Z.) connexa Berlese, 1904 & & & + & & \\
\hline 40 & Oribatula (Z.) undulata Berlese, $1916^{\text {म }}$ & + & + & & + & + \\
\hline 41 & Hemileius (Simkinia) ovalis Kulijev, 1968 & + & & & & \\
\hline 42 & Hemileius (Urubambates) elongatus (Krivolutsky, 1969) Кд & & & & & + \\
\hline 43 & Scheloribates barbatulus Mihelčič, $1956^{\text {म }}$ & + & & & & \\
\hline 44 & Scheloribates laevigatus laevigatus (Koch, 1835) & + & & & & \\
\hline 45 & Scheloribates pallidulus latipes Koch, 1844) & & & + & & \\
\hline 46 & Protoribates (P.) capucinus Berlese, 1908 & + & & + & & \\
\hline 47 & Peloribates perezinigoi Shtancaeva, Grikurova, Subias, $2011^{\mathrm{K}} \mathrm{A}$ & + & + & & + & \\
\hline 48 & Galumna lanceata (Oudemans, 1900) & + & & & & + \\
\hline 49 & Psammogalumna sp. ${ }^{\mathrm{K} \mathrm{L}^{* * *}}$ & & & + & & \\
\hline & Итого видов: & 26 & 17 & 18 & 3 & 8 \\
\hline
\end{tabular}

Примечание: $\quad$ к-виды, впервые отмеченные на Кавказе (12);

Д - виды, впервые отмеченные для Дагестана (20);

*** - новый и предположительно новый для науки виды (2).

Всего обнаружено 49 видов орибатид, относящихся к 39 родам и подродам и 24 семействам (табл. 1), в том числе 20 видов впервые отмечены для фауны Дагестана, а 12 видов для Кавказа. Описан новый для науки вид Oribatula (Zygoribatula) caspica Shtanchaeva, Grikurova, Subias, 2011 [2], обнаружен предположительно новый для науки вид - Psammogalumna sp.

Ареалогия видов орибатид (табл. 2) указана согласно каталогам орибатид Кавказа и мира [5; 6; 7]. 
Зоогеографическая характеристика панцирных клещей прибрежных экосистем и островов северо-западной части Каспийского моря

\begin{tabular}{|c|c|c|c|c|c|c|c|c|c|c|}
\hline \multirow[b]{2}{*}{ № } & \multirow[b]{2}{*}{ Виды панцирных клещей } & \multicolumn{9}{|c|}{ Зоогеографические группы } \\
\hline & & 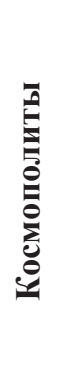 & 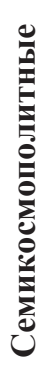 & 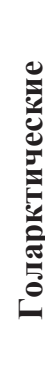 & 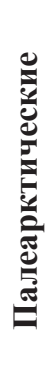 & 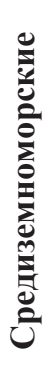 & 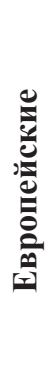 & 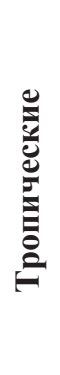 & 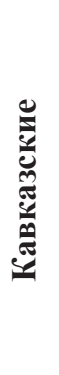 & 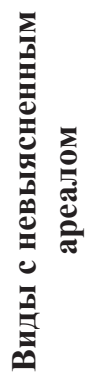 \\
\hline 1 & Aphelacarus acarinus (Berlese, 1910) & & + & & & & & & & \\
\hline 2 & $\begin{array}{l}\text { Haplochthonius (H.) sanctaeluciae Bernini, } \\
1973\end{array}$ & & & & + & & & & & \\
\hline 3 & $\begin{array}{l}\text { Sphaerochthonius pallidus Muñoz- } \\
\text { Mingarro, } 1987\end{array}$ & & & & & + & & & & \\
\hline 4 & $\begin{array}{l}\text { Sphaerochthonius splendidus (Berlese, } \\
\text { 1904) }\end{array}$ & & & & & & & + & & \\
\hline 5 & $\begin{array}{c}\text { Epilohmannia cylindrica cylindrica } \\
\text { (Berlese, 1904) }\end{array}$ & + & & & & & & & & \\
\hline 6 & $\begin{array}{c}\text { Epilohmannia cylindrica minima } \\
\text { Schuster, } 1960\end{array}$ & & & & & & + & & & \\
\hline 7 & $\begin{array}{c}\text { Papillacarus pseudoaciculatus } \\
\text { Mahunka, } 1980\end{array}$ & & & & & + & & & & \\
\hline 8 & Neoliodes ionicus Sellnick, 1931 & & & & & + & & & & \\
\hline 9 & $\begin{array}{c}\text { Pyroppia tajikistanica Krivolutsky et } \\
\text { Christov, } 1970\end{array}$ & & & & + & & & & & \\
\hline 10 & $\begin{array}{l}\text { Ramusella (R.) puertomonttensis Hammer, } \\
1962\end{array}$ & & & & & & & + & & \\
\hline 11 & $\begin{array}{l}\text { Ramusella (Rectoppia) strinatii (Mahunka, } \\
\text { 1980) }\end{array}$ & & & & & + & & & & \\
\hline 12 & $\begin{array}{l}\text { Discoppia (Cylindroppia) cylindrica } \\
\text { (Pérez-Íñigo, 1965) }\end{array}$ & & & & + & & & & & \\
\hline 13 & Microppia minus minus (Paoli, 1908) & + & & & & & & & & \\
\hline 14 & $\begin{array}{l}\text { Lauroppia similifallax Subías et Mínguez, } \\
1986\end{array}$ & & & & & & + & & & \\
\hline 15 & Oppiella (O.) nova nova (Oudemans, 1902) & + & & & & & & & & \\
\hline 16 & $\begin{array}{l}\text { Subiasella (Lalmoppia) subiasi (Mahunka, } \\
\text { 1987) }\end{array}$ & & & & & & + & & & \\
\hline 17 & $\begin{array}{c}\text { Suctobelbella (S.) acutidens duplex } \\
\text { (Strenzke, 1950) }\end{array}$ & & & + & & & & & & \\
\hline 18 & $\begin{array}{l}\text { Suctobelbella (S.) subcornigera } \\
\text { subcornigera (Forsslund, 1941) }\end{array}$ & & + & & & & & & & \\
\hline 19 & $\begin{array}{l}\text { Suctobelbella (Flagrosuctobelba) nasalis } \\
\text { (Forsslund, 1941) }\end{array}$ & & & & + & & & & & \\
\hline 20 & $\begin{array}{l}\text { Tectocepheus velatus sarekensis Trägårdh, } \\
1910\end{array}$ & + & & & & & & & & \\
\hline 21 & Scutovertex sculptus Michael, 1879 & & & & + & & & & & \\
\hline 22 & $\begin{array}{l}\text { Bipassalozetes (Passalobates) linearis } \\
\text { (Higgins et Woolley, 1962) }\end{array}$ & & & + & & & & & & \\
\hline 23 & Passalozetes africanus Grandjean, 1932 & & & & + & & & & & \\
\hline
\end{tabular}




\begin{tabular}{|c|c|c|c|c|c|c|c|c|c|c|}
\hline 24 & Berlesezetes ornatissimus (Berlese, 1913) & & & & & & & + & & \\
\hline 25 & $\begin{array}{l}\text { Oribatella (Oribatella) reticulata } \\
\text { Berlese, } 1916 .\end{array}$ & & & + & & & & & & \\
\hline 26 & $\begin{array}{l}\text { Oribatella (O.) tridactyla Ruiz, Subías et } \\
\text { Kahwash, } 1991\end{array}$ & & & & & + & & & & \\
\hline 27 & Tectoribates ornatus (Schuster, 1958) & & & & + & & & & & \\
\hline 28 & $\begin{array}{l}\text { Trichoribates (Trichoribates) novus (Sell- } \\
\text { nick,1928) }\end{array}$ & & & + & & & & & & \\
\hline 29 & $\begin{array}{l}\text { Trichoribates (Latilamellobates) clavatus } \\
\text { Mihelčič, } 1956\end{array}$ & & & & & + & & & & \\
\hline 30 & $\begin{array}{l}\text { Trichoribates (Latilamellobates) naltschicki } \\
\text { (Shaldybina, 1971) }\end{array}$ & & & & & + & & & & \\
\hline 31 & $\begin{array}{l}\text { Zetomimus (Protozetomimus) acutirostris } \\
\text { (Mihelčič, 1957) }\end{array}$ & & & & & + & & & & \\
\hline 32 & $\begin{array}{l}\text { Chamobates (Xiphobates) rastratus (Hull, } \\
\text { 1914) }\end{array}$ & & & & + & & & & & \\
\hline 33 & $\begin{array}{l}\text { Punctoribates (Punctoribates) punctum } \\
\text { (Koch,1839) }\end{array}$ & & + & & & & & & & \\
\hline 34 & $\begin{array}{l}\text { Punctoribates (Minguezetes) hexagonus } \\
\text { Berlese, } 1908\end{array}$ & & & + & & & & & & \\
\hline 35 & Podoribates longipes (Berlese, 1887) & & & + & & & & & & \\
\hline 36 & $\begin{array}{l}\text { Oribatula (Oribatula) interrupta interrupta } \\
\text { (Willmann, 1939) }\end{array}$ & & & + & & & & & & \\
\hline 37 & $\begin{array}{l}\text { Oribatula (O.) tibialis saljanica Kulijev, } \\
1962\end{array}$ & & & & & & & & + & \\
\hline 38 & $\begin{array}{l}\text { Oribatula (Zygoribatula) caspica } \\
\text { Shtancaeva, Grikurova, Subias, } 2011\end{array}$ & & & & & & & & + & \\
\hline 39 & Oribatula (Z.) connexa Berlese, 1904 & & & & & & & + & & \\
\hline 40 & Oribatula (Z.) undulata Berlese, $1916^{Д}$ & & & & & & & + & & \\
\hline 41 & Hemileius (Simkinia) ovalis Kulijev, 1968 & & & & & + & & & & \\
\hline 42 & $\begin{array}{l}\text { Hemileius (Urubambates) elongatus (Kri- } \\
\text { volutsky, 1969) }\end{array}$ & & & & + & & & & & \\
\hline 43 & Scheloribates barbatulus Mihelčič, 1956 & & & & + & & & & & \\
\hline 44 & $\begin{array}{l}\text { Scheloribates laevigatus laevigatus (Koch, } \\
1835 \text { ) }\end{array}$ & & + & & & & & & & \\
\hline 45 & $\begin{array}{l}\text { Scheloribates pallidulus latipes Koch, } \\
1844)\end{array}$ & & & + & & & & & & \\
\hline 46 & Protoribates (P.) capucinus Berlese, 1908 & + & & & & & & & & \\
\hline 47 & $\begin{array}{l}\text { Peloribates perezinigoi Shtanchaeva, } \\
\text { Grikurova, Subias, } 2011\end{array}$ & & & & & + & & & & \\
\hline 48 & Galumna lanceata (Oudemans, 1900) & & & & + & & & & & \\
\hline 49 & Psammogalumna sp. & & & & & & & & & + \\
\hline & Итого видов: & 5 & 4 & 8 & 11 & 10 & 3 & 5 & 2 & 1 \\
\hline
\end{tabular}

Наибольшую долю в спектре фаунистических элементов составляют виды с палеарктическим (23\%) и средиземноморским (21\%) ареалами, к первой группе относятся 11 видов (Haplochthonius (H.) sanctaeluciae, Pyroppia tajikistanica, Discoppia (Cylindroppia) cylindrica, Suctobelbella (Flagrosuctobelba) nasalis, Scutovertex sculptus, Passalozetes africanus, Tectoribates ornatus, Chamobates (Xiphobates) rastratus, Hemileius (Urubambates) elongates, Scheloribates barbatulus, Galumna lanceata), ко второй - 10 видов (Sphaerochthonius pallidus, Papillacarus pseudoaciculatus, Neoliodes ionicus, Ramusella (Rectoppia) strinatii, Oribatella (O.) tridactyla, Trichoribates (Latilamellobates) clavatus, Trichoribates (Latilamellobates) naltschicki, Zetomimus (Protozetomimus) acutirostris, Hemileius (Simkinia) ovalis, Peloribates perezinigoi).

Довольно широко представлены голаркты, к ним относятся 8 видов (Suctobelbella (S.) acutidens duplex, Bipassalozetes (Passalobates) linearis, Oribatella (O.) reticulata, Trichoribates (T.) novus, Punctoribates (Minguezetes) hexagonus, Podoribates longipes, Oribatula (O.) interrupta interrupta, Scheloribates pallidulus latipes), которые составляют 16\% от общего их числа. Космополиты (5 видов: Epilohmannia cylindrica cylindrica, Microppia minus minus, Oppiella (O.) nova nova, Tectocepheus velatus sarekensis, Protoribates (P.) capucinus) и ce- 
микосмополиты (4 вида: Aphelacarus acarinus, Suctobelbella (S.) subcornigera subcornigera, Punctoribates (P.) punctum, Scheloribates laevigatus laevigatus) составляют 10\% и $8 \%$ соответственно.

Виды с тропическим ареалом (5 видов) составляют 10\% от общего числа (Sphaerochthonius splendidus, Ramusella (R.) puertomonttensis, Berlesezetes ornatissimus, Oribatula (Zygoribatula) connexa, Oribatula (Z.) undulata). Виды с кавказским распространением (O. (O.) tibialis saljanica и Oribatula (Z.) caspica) составляют $4 \%$. сунке 1 .

Предположительно новый вид (Psammogalumna sp.) отнесен пока к видам с невыясненным ареалом.

Соотношение зоогеографических групп панцирных клещей исследованной территории показано на ри-

Рисунок 1

Соотношение фаунистических групп панцирных клещей побережья и островов северо-западной части Каспийского моря

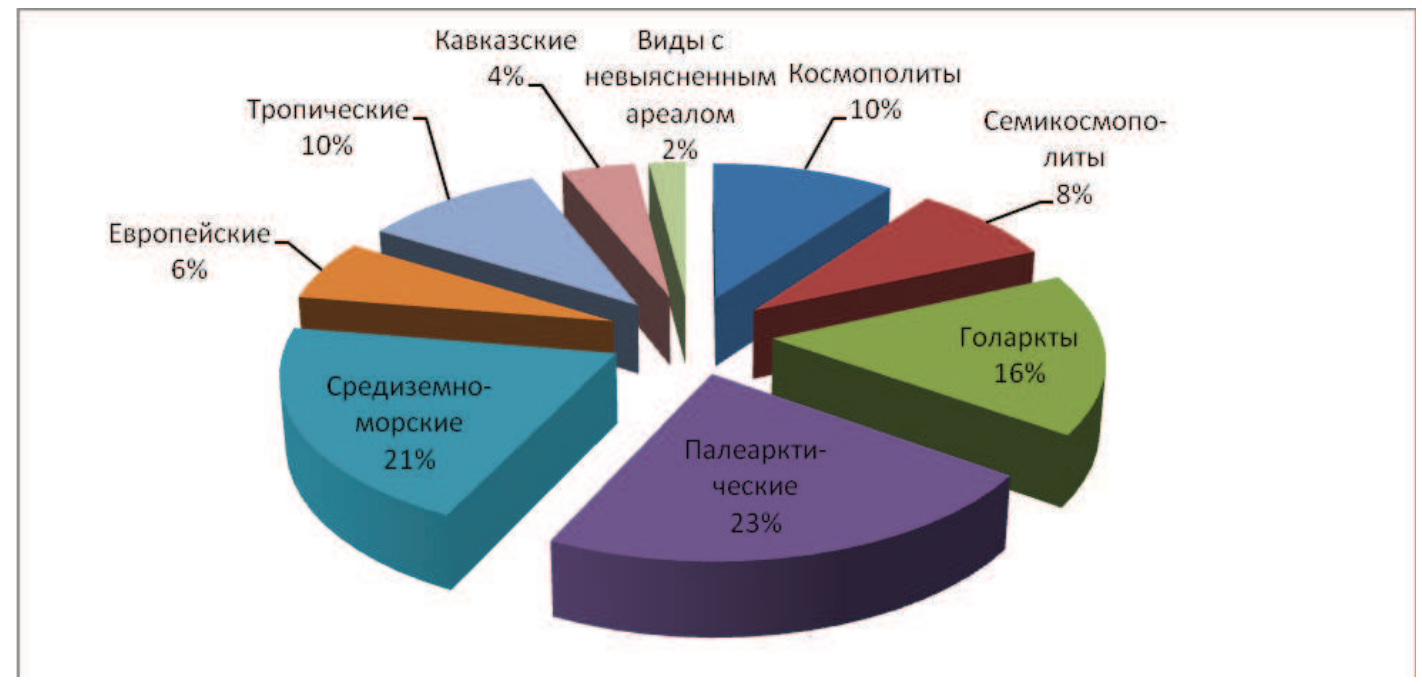

\section{Библиографический список}

1. Абдурахманов Г.М., Грикурова А.А., Штанчаева У.Я., Субиас Л.С. Фауна панцирных клещей (Acariformes,Oribatida) прибрежных экосистем и островов Северо-Западного Каспия и их жизненные формы // Юг России. 2011. № 2. С. 2429.

2. Штанчаева У.Я., Грикурова А.А., Субиас Л.С. Панцирные клещи (Acariformes,Oribatida) побережья и островов Каспийского моря // Зоол. журнал. 2011. Т. 90, № 10. С. 1175-1179.

3. Муртузалиев Р.А. Карта фрлористических районов Дагестана // Материалы VI международной конференции «Биологическое разнообразие Кавказа». Нальчик: КБГУ, 2004. С. 187-188.

4. Чиликина Л.Н., Волкова И.И., Ярулина Н.А., Шифферс Е.В. Карта растительности Дагестанской АССР. Под редакцией Е.В. Шифферс. М.: Наука, 1960.

5. Штанчаева У.Я., Субиас Л.С. Каталог панцирных клещей Кавказа. Махачкала: ДНЦ РАН, 2010. 276 с.

6. Subias L.S., Shtanchaeva U.Ya., Arillo A. Listado de los ácaros oribátidos (Acariformes: Oribatida) de las diferentes regiones biogeográficas del mundo // www.sea-entomologia.org. Monografia electronica de Sociedad Entomologica Aragonesa. Zaragoza, 2012. 31-12-2012. $815 p$.

7. Subías L.S. Listado sistemático, sinonímico y biogeográfico de los ácaros oribátidos (Acariformes, Oribatida) del mundo (1758-2002) // Graellsia. № 60. Numero extraordinario, 2004. Pp. 3-305. - Listado sistemático, sinonímico y biogeográfico de los ácaros oribátidos (Acariformes, Oribatida) del mundo (excepto fósiles). Online versión actualizado en junio de 2006, en abril de 2007, en mayo de 2008, en abril de 2009, en julio de 2010, en febrero de 2011 y en febrero de 2012. 564p. //http://www.ucm.es/info/zoo/Artropodos/Catalogo.pdf

\section{Bibliography}

1. Abdurahmanov G.M., Grikurova A.A., Shtanchaeva U.Ya., Subias L.S. Fauna oribatid mites (Acariformes, Oribatida) coastal ecosistems and islands of the Nort-West of the Caspian Sea and their forms of life. // South of Russia, 2011. №. 2. P. 24-29. 
2. Shtanchaeva U.Ya., Grikurova A.A., Subias L.S. Oribatid mites (Acariformes, Oribatida) of coast and islands of Caspian Sea // Zool. journ., 2011. Vol. 90. № 10. Pp. 1175-1179.

3. Murtuzaliev R.A. Map of floristic regions of Dagestan. Materials of the VI International Conference "Biodiversity of the Caucasus." Nalchik: KBGU, 2004. Pp. 187-188.

4. Chilikina L.N., Volkova I.I., Yarullina N.A., Shiffers E.V. Vegetation map of Daghestan. Editor-shif E.V. Shiffers, 1960.

5. Shtanchaeva U.Ya., Subias L.S. Catalog oribatid mites of the Caucasus. Makhachkala: Dagestan Scientific Center, RAS, 2010. Pp. 124-228.

6. Subias L.S., Shtanchaeva U.Ya., Arillo A. Listado de los ácaros oribátidos (Acariformes: Oribatida) de las diferentes regiones biogeográficas del mundo // www.sea-entomologia.org. Monografia electronica de Sociedad Entomologica Aragonesa. Zaragoza, 2012. 31-12-2012. 815 p.

7. Subías L.S. Listado sistemático, sinonímico y biogeográfico de los ácaros oribátidos (Acariformes, Oribatida) del mundo (1758-2002) // Graellsia. № 60. Numero extraordinario, 2004. Pp. 3-305. - Listado sistemático, sinonímico y biogeográfico de los ácaros oribátidos (Acariformes, Oribatida) del mundo (excepto fósiles). Online versión actualizado en junio de 2006, en abril de 2007, en mayo de 2008, en abril de 2009, en julio de 2010, en febrero de 2011 y en febrero de 2012. 564p. //http://www.ucm.es/info/zoo/Artropodos/Catalogo.pdf

УДК $591.9(470.67)$

\title{
ОБЪЕМЫ РОДОВ И ВИДОВОЙ СОСТАВ ОРИБАТИД (ACARIFORMЕS, ORIBATIDA) ВНУТРЕННЕГО ГОРНОГО ДАГЕСТАНА
}

๑ 2013 Абдурахманов Г.М., Давудова Э.3. Дагестанский государственный университет

\begin{abstract}
Данная работа основана на исследованиях и материалах с 2000 по 2012 годы, с применением метода отбора почвенных проб, согласно методике количественных учетов [3-5]. В результате проведенных исследований на территории Внутреннего горного Дагестана выявлено 190 видов орибатид, относящиеся к 83 родам.

This work is based on research and materials collected from 2000 to 2012, with the application of the method of selection of soil samples, according to the methodology of the quantitative survey. As a result of the conducted researches on the territory of the internal mountain Dagestan found 190 species of Oribatida belonging to 83 generations.

Ключевые слова: Внутреннегорный Дагестан, фауна, орибатиды, роды, виды.

Key words: Internal mountain Dagestan, fauna, Oribatida,genus, species.
\end{abstract}

Республика Дагестан - уникальная и своеобразная территория Российской Федерации. Многообразен ее рельеф и не менее многообразна флора и фауна. Горная часть занимает 56\% от общей площади [3]. Исторически сложившиеся и современные физико-географические и экологические условия определили богатство, большое видовое разнообразие, высокую численность фауны и мозаичность ее происхождения. Панцирные клещи являются одной из основных групп почвенной микрофауны. Изучение фауны Внутреннего Горного Дагестана имеет большое значение для познания биразнообразия Кавказа в целом [2]. Литература по систематике, фаунистике и экологии насекомых, многоножек, паукообразных насчитывает тысячи названий. Тем не менее, фауну даже ведущих групп беспозвоночных до сих пор нельзя считать удовлетворительно изученной.

Данная работа основана на исследованиях и материалах с 2000 по 2012 годы, с применением метода отбора почвенных проб, согласно методике количественных учетов [4-6]. В результате проведенных исследований на территории Внутреннего горного Дагестана выявлено 190 видов орибатид, относящиеся к 83 родам.

Надсемейство Hypochthonioidea Berlese, 1910

В мировой фауне представлено 3 семействами [8], на Кавказе [6] и на исследуемой территории - 2 семействами.

Семейство Нуросhthoniidae Berlese, 1910

По миру включает 4 рода и 1 подрод, по Кавказу - 2 рода, для исследуемой территории характерен 1 род.

\section{Pод Hypochthonius Koch, 1835}

Космополит (исключая Эфиопскую область и Антарктику), включающий 6 видов и 3 подвида. На Кавказе и в исследуемой территории отмечены 2 вида - H. luteus Oudemans, 1917 (=Hypochthonius luteus rectosetosus Krivolutsky, 1965); H. rufulus Koch, 1835 (=Hypochthonius rufulus brevisetosus Krivolutsky, 1965) (=Hypochthonius 